\title{
Networked Privacy Beyond the Individual: Four Perspectives to 'Sharing'
}

\author{
Airi Lampinen \\ Mobile Life Centre, \\ Stockholm University \\ Stockholm, Sweden \\ airi@mobilelifecentre.org
}

\begin{abstract}
Synthesizing prior work, this paper provides conceptual grounding for understanding the dialectic of challenges and opportunities that social network sites present to social life. With the help of the framework of interpersonal boundary regulation, this paper casts privacy as something people do, together, instead of depicting it as a characteristic or a possession. I illustrate interpersonal aspects of networked privacy by outlining four perspectives to 'sharing'. These perspectives call for a rethink of networked privacy beyond an individual's online endeavors.
\end{abstract}

\section{Author Keywords}

Privacy; interpersonal boundary regulation; social network site; Facebook; Last.fm; Couchsurfing

\section{ACM Classification Keywords}

H.5.m. Information interfaces and presentation (e.g., HCI): Miscellaneous.

\section{INTRODUCTION}

Social network sites (SNSs), such as Facebook, MySpace, LinkedIn, and Instagram, present people with novel opportunities to maintain social ties; craft an online presence; and, as a result, gain access to social validation and meaningful feedback [14]. As these platforms have become part of the everyday life for millions of people, severe concerns have been raised regarding their implications for social interaction and societies at large. This paper provides grounding for understanding the dialectic of challenges and opportunities that social network sites present to daily social life by building on an understanding of networked privacy that has been developed in and around Human-Computer Interaction over the past decade (for early work, see e.g. [10]).

It is worth noting that the nature of privacy is a complex and long-standing question that has attracted the attention of scholars in several disciplines already well before the

Copyright $(\mathrm{C} 2015$ is held by the author(s). Publication rights licensed to Aarhus University and ACM

5th Decennial Aarhus Conference on Critical Alternatives

August 17-21, 2015, Aarhus Denmark

DOI: http://dx.doi.org/10.7146/aahcc.v1i1.21300 rise of mainstream social media (for a review, see [8]). Rather than attempting a general discussion of privacy, this paper narrows in on a more limited set of issues by addressing interpersonal aspects of networked privacy.

In the following, I deploy the framework of interpersonal boundary regulation [1] in order to approach privacy as something people do together, i.e. a collaborative activity, instead of depicting it as a characteristic of a piece of content or a possession of an individual. I provide examples of how the negotiation of accessibility and inaccessibility that characterizes social relationships plays out in the context of social network sites by outlining four perspectives to 'sharing' that have emerged from prior empirical studies. These perspectives call for a rethink of privacy in our networked age beyond the individual and his/her online endeavors.

\section{SOCIAL NETWORK SITES}

Ellison and boyd [4, p. 158] characterize a social network site as 'a networked communication platform in which participants (1) have uniquely identifiable profiles that consist of user-supplied content, content provided by other users, and/or system-provided data; (2) can publicly articulate connections that can be viewed and traversed by others; and (3) can consume, produce, and/or interact with streams of user-generated content provided by their connections on the site.' Nowadays, social network sites are commonly accessed through a variety of devices and platforms, such as applications on mobile devices. People interact with social network sites not only when they access them directly; rather, they may come across integrated features almost anywhere. For instance, Facebook's 'Like' feature, which allows people to indicate with a click their approval and enjoyment of pieces of content, is now nearly ubiquitous on the Web.

The widespread adoption of social network sites into everyday interaction affects sociality more broadly than just in terms of the activities that take place on these platforms. Ubiquitous access to social network sites and participation in social interaction via them is of course not universal. However, social network sites have become a pervasive part of social life for many and, at least in large parts of the Western world, even those who do not use them in their day-to-day life are embedded in social settings that are shaped by their existence. Typically even those who opt out 
of using SNSs have, and indeed must have, an opinion on them. Finally, refusing to join a social network site does not mean that one would not be referred to or featured in the service (for instance, in photos or textual anecdotes that others share).

\section{INTERPERSONAL BOUNDARY REGULATION}

The framework of boundary regulation settings [1] was first introduced by social psychologist Irwin Altman in the 1970s. Over the past decades, it has been developed further, most prominently as Communication Privacy Management theory $[11,12]$. Interpersonal boundary regulation refers to the negotiation of accessibility and inaccessibility that characterizes social relationships [2]. Interpersonal boundary regulation is a core process of social life: Interpersonal boundaries are constantly regulated through negotiations that draw lines of division between self and others, and 'us' versus 'them'. They are used to structure how and with whom people interact. When successful, interpersonal boundary regulation allows people to come to terms with who they are and how they relate with one another. In contrast, less successful or failing efforts to regulate boundaries are experienced as conflict, confusion, and clashes in expectations, that is, as boundary turbulence [11] that necessitates re-negotiating rules of regulation.

As individuals, groups, and societies, we regulate access to social interaction both by how physical spaces are built and through the behaviors that take place in them. Boundary regulation practices abound from making or avoiding eye contact to closing or opening a door. Boundaries of professional and leisure life are negotiated in intricate ways that range from what people wear and eat to with whom, about what, and in what kind of language they converse [9]. These practices are applied to achieve contextually desirable degrees of social interaction as well as to build and sustain relations with others and with the self.

While interpersonal boundaries are not just an analogue of physical demarcations, they are, in part, determined by physical structures. For instance, walls, doors, windows, and furniture shape access to various spaces and to the social interaction that can take place in them. Access to visual and auditory information is limited by what is physically possible [3]: Only people within a restricted geographical radius at any given time can see and hear what is being said and done by co-present others. Those witnessing an incident in an unmediated situation can tell others about it, carrying it further, but there are only so many people who can observe an event first-hand. Sharing one's experiences and observations with people who are not present requires effort. Moreover, in face-to-face situations, those doing and saying things typically have a fairly good sense of who can see and hear their actions, as they can observe their audience in a shared temporal and spatial setting. Someone could, of course, be eavesdropping out of sight, but having such an unintended audience would be exceptional and violate commonly held rules of decorum.
The widespread adoption of SNSs challenges customary mechanisms for regulating interpersonal boundaries. For instance, when it comes to social interaction via these services, it is not reasonable to rely solely on the supportive structures that time and space can provide. In 2003, Palen and Dourish [10] paved the way for ongoing research into interpersonal networked privacy by raising the issue of boundary regulation in an increasingly networked world. While the boundary regulation framework originates in Altman's considerations of social life in physical settings [1], offline activities have often been bypassed when examining boundary regulation in the context of SNSs. Yet, the social interaction that takes place in SNSs is embedded in the wider fabric of social relationships that surround individuals. Boundary regulation efforts must therefore be understood holistically, since the broad adoption of these platforms weaves them tightly into everyday life, with consequences also to those who can or will not use them.

Finally, the idea that not just individuals, but groups, too, need to regulate their boundaries was a part of Altman's [1] original characterization of privacy as boundary regulation. Yet, this notion of group privacy has received scarce scholarly attention within HCI, even though Petronio and colleagues $[11,12]$ have elaborated explicitly on how individuals and groups make decisions over revealing or concealing private information, proposing that individuals develop and use rules collaboratively to control information flows. In contrast to Altman whose work focuses mainly on personal boundaries, Petronio pays particular attention to how people manage collectively co-owned information and collective boundaries, such as those within families and groups of friends. Both Altman and Petronio suggest that individuals need to regulate interpersonal boundaries proactively in order to achieve a desired degree of privacy.

\section{FOUR PERSPECTIVES TO 'SHARING'}

This section illustrates different interpersonal aspects of networked privacy by outlining four perspectives to 'sharing' that takes place in the context of SNSs. The notion of 'sharing' is grounded in prior empirical work on networked privacy $[5,6,7,13]$ where it has emerged as a central way in which people talk about their engagement with SNSs and their efforts to manage what gets published, to whom, and with what kinds of implications. I draw on prior qualitative, interpretative analyses of ongoing sensemaking surrounding social life in the context of SNSs and of the emerging practices for regulating interpersonal boundaries in settings wherein (1) people may share content with multiple groups at once, (2) others may share content on their behalf, (3) sharing can be done via automated mechanisms, and (4) people may share as a group. Instead of treating 'sharing' as a monolithic, well-defined activity, I consider and contrast the sharing of manually selected digital content; the streaming of automatically tracked behavioral information; and, acts of sharing that directly challenge the hypothetical online-offline divide. 


\section{Sharing with Multiple Groups}

Social network sites may bring about group co-presence, a situation in which many groups important to an individual are simultaneously present in one context and their presence is salient for the individual [6]. Group co-presence makes it difficult to keep traditionally separate social spheres apart from one another. Maintaining a broad social network in an SNS may lead to a sense of having to present oneself and one's social connections consistently with everyone. In an early study [6], participants reported few social tensions related to group co-presence on Facebook per se, but the authors argue that group co-presence was unproblematic only insofar as it was made unproblematic with the aid of behavioral and mental practices. The challenges related to this tendency of SNSs to flatten diverse audiences into a single group have since been well documented, often under the rubric of context collapse (for an overview, see [16]). A central challenge in sharing with multiple groups at once lies in regulating interpersonal boundaries in a situation where it is hard to keep track of to whom access is provided, or to figure out whether or when these audiences interact with content that is made available to them.

\section{Sharing on Behalf of Others}

While individuals can regulate interpersonal boundaries on their own, ultimately their success always relies on others' support of their efforts to draw boundaries in a certain way. Interpersonal boundary regulation is best understood as cooperative on two levels: First, cooperation is manifested in continuous, subtle acts of contesting or supporting others' efforts: maintaining a boundary or making a successful claim to identity requires that others affirm one's actions and support the definition of oneself that is put forward. Lampinen et al. [7] report on Facebook users reckoning on how and by whom the content that they shared would be viewed and interpreted. Some pondered whether the content they were sharing might inadvertently create challenges for a friend. Participants' efforts to cooperate included considerate acts of sharing, discretion in self-censorship, and benevolent interpretation. Second, in response to the need to deal with content disclosed by others and with one's power to share on behalf of others, individuals cooperated occasionally more explicitly, too. This involved coming into agreement on shared codes of conduct through explicit negotiation of what to share, with whom, and under what conditions [7]. While interpersonal boundary regulation was not readily discussed as explicit cooperation, participants placed a strong emphasis on being trustworthy and considerate [7]. Indicating a willingness to take others into consideration and an awareness of reliance on others' consideration, these participants saw this was not only as something desired of others but also as a standard they strove to live up to.

\section{Sharing via Automated Mechanisms}

Providing a contrast to the aforementioned examples of manual sharing on Facebook, the music-centered Last.fm, where the main content that is being shared is information about music listening, is an instance of a class of services that allow users to stream behavioral data via automated sharing mechanisms. Automated sharing mechanisms are promoted as a means to make sharing increasingly effortless and authentic in its presumed completeness, but research indicates that much work can go into regulating what is being shared via them [13]. Interpersonal boundary regulation efforts in the presence of automated sharing mechanisms entail decisions that extend beyond choices over sharing per se and affect, instead, how participants behave in the first place: Uski \& Lampinen [15] note that while norms concerning manual sharing on Facebook focus mainly on what and how one should share, the norms sanctioning automated sharing on Last.fm target primarily music listening, that is, the 'actual' behavior. In other words, when it comes to interpersonal boundary regulation in the presence of automated sharing, there are, first, interpersonal boundary regulation practices that regulate what is publicized, and second, efforts to regulate what to do and, consequently, what kind of data will be available for sharing later on. These efforts show how users' agency to regulate interpersonal boundaries is restricted neither to adjusting the privacy settings provided within an SNS nor to selecting from among the choices services propose to their users in the user interface. Finally, it is worth noting that users engage in efforts to regulate sharing not only when the sharing mechanism risks publicity of content that they do not want to have in their profiles but also when the sharing mechanism fails to publicize content that it should have and that users want to appear in their profiles $[13,15]$. This illustrates well how boundary regulation is not solely a matter of restricting access but also one of providing it.

\section{Sharing as a Group}

Shifting the focus further beyond mainstream social network sites, consider Couchsurfing, a service where members can engage in hospitality exchange by hosting visitors or by staying with others as guests. In a recent study Lampinen [5] examined how multi-person households share accounts and regulate access to their domestic sphere as they welcome 'couchsurfers'. Profiles on Couchsurfing serve to present two interwoven but distinguishable aspects of households: the domestic space and the people who live in it. The study touches upon group privacy by illustrating how, beyond sharing credentials, account sharing involves complex negotiations over how to present multiple people in a single profile, how to coordinate the households' outfacing communication and decisions over whether to grant potential visitors access to the home, as well as how to share the benefits of a good reputation in a fair way. Here, sharing as a group entails multi-faceted boundary regulation that takes place not only in online interaction but also in the course of interacting with guests face-to-face. Thus, the study challenges the hypothetical online-offline divide with its focus on the practice of hosting where 'sharing' online and offline are tightly interwoven. 


\section{CONCLUSION}

Although social network sites have characteristics that disrupt conventional premises of interpersonal boundary regulation, boundaries are regulated through co-operative processes also in their presence. In fact, the use of social network sites may even make the performative nature of social life more visible than is usual or desirable. Increased awareness of the work that goes into achieving appropriate social interaction and sustaining meaningful relationships may feel uncomfortable as it challenges the illusion of the effortless authenticity of everyday socializing.

This paper invites us to consider the limitations inherent in conceiving of privacy management as an individual-level endeavor to do with control over information by synthesizing research that illustrates how people regulate both individual and collective boundaries in cooperation with one other, with consideration to others, and in line with the affordances of different technologies and relevant social norms. When the tools and features of a service create constraints in day-to-day life, people may opt to lessen their engagement with the service $[6,7]$ or to change the way they behave in order to manage what is being shared $[7,13]$.

To design technologies and policies that are supportive of people's boundary regulation aims and practices in an increasingly networked world, we first need to understand those pursuits and the reasoning behind them. By highlighting the cooperative nature of these everyday efforts to 'make the world work', this paper calls for a rethink of networked privacy beyond the individual and his/her online endeavors.

\section{ACKNOWLEDGMENTS}

This work has been supported by the Mobile Life VINN Excellence Centre. I thank my colleagues at Mobile Life, University of Helsinki, Helsinki Institute for Information Technology HIIT, University of California, Berkeley, and Microsoft Research New England.

\section{REFERENCES}

1. Altman, I. (1975). The environment and social behavior: Privacy, personal space, territory, crowding. Monterey, CA: Brooks/Cole Pub. Co.

2. Altman, I., \& Gauvain, M. (1981). A cross-cultural and dialectic analysis of homes. In Spatial representation behavior across the life span: Theory and application. New York: Academic Press.

3. boyd, d. (2008). Why youth $\bullet$ social network sites: The role of networked publics in teenage social life. In D. Buckingham (ed.), Youth, identity, and digital media (pp. 119-142). Cambridge, MA: MIT Press.
4. Ellison, N. B., \& boyd, D. M. (2013). Sociality through social network sites. In W. H. Dutton (ed.), The Oxford handbook of Internet studies (pp. 151-172). Oxford University Press.

5. Lampinen, A. (2014). Account Sharing in the Context of Networked Hospitality Exchange. In Proceedings of the 2014 Conference on Computer-Supported Cooperative Work (pp. 499-501). ACM.

6. Lampinen, A., Tamminen, S., \& Oulasvirta, A. (2009). All my people right here, right now: Management of group co-presence on a social networking site. In Proceedings of the 2009 International Conference on Supporting Group Work (pp. 281-290). ACM.

7. Lampinen, A., Lehtinen, V., Lehmuskallio A., \& Tamminen, S. (2011). We're in it together: Interpersonal management of disclosure in social network services. In Proceedings of the 2011 Annual Conference on Human Factors in Computing Systems (pp. 3217-3226). ACM.

8. Newell, P. B. (1995). Perspectives on privacy. Journal of Environmental Psychology, 15(2), 87-104.

9. Nippert-Eng, C. E. (1996). Home and work: Negotiating boundaries through everyday life. Chicago: University of Chicago Press.

10. Palen, L., \& Dourish, P. (2003). Unpacking privacy for a networked world. In Proceedings of the SIGCHI conference on Human factors in computing systems (pp. 129-136). ACM.

11. Petronio, S. S. (2002). Boundaries of privacy: Dialectics of disclosure. Albany, NY: State University of New York Press.

12. Petronio, S. (2013). Brief status report on communication privacy management theory. Journal of Family Communication, 13(1), 6-14.

13. Silfverberg, S., Liikkanen, L.A., \& Lampinen, A. (2011). 'I'll press play, but I won’t listen': Profile work in a music-focused social network service. In Proceedings of the 2011 Conference on ComputerSupported Cooperative Work (pp. 207-216). ACM.

14.Stern, S. (2008). Producing sites, exploring identities: Youth online authorship. In D. Buckingham (ed.), Youth, identity, and digital media (pp. 95-117). Cambridge, MA: MIT Press.

15.Uski, S., \& Lampinen, A. (2014). Social norms and selfpresentation on social network sites: Profile work in action. New Media \& Society.

16. Vitak, J. (2012). The impact of context collapse and privacy on social network site disclosures. Journal of Broadcasting \& Electronic Media, 56(4), 451-470. 